\title{
Nuclear Stopping as A Probe to In-Medium Nucleon-Nucleon Cross Section in Intermediate Energy Heavy Ion Collisions
}

\author{
Jian-Ye Liu ${ }^{1,2,3}$, Wen-Jun Guo ${ }^{2}$, Shun-Jin Wang ${ }^{1,3,4}$ \\ Wei Zuo ${ }^{1,2,3}$, Qiang Zhao ${ }^{2}$, Yan-Fang Yang ${ }^{2}$ \\ ${ }^{1}$ Center of Theoretical Nuclear Physics, National Laboratory of Heavy \\ Ion Accelerator, Lanzhou 730000, P. R. China \\ ${ }^{2}$ Institute of Modern Physics, Chinese Academy of Sciences, Lanzhou 730000, P. R. China \\ ${ }^{3}$ CCAST (World Lab.), P.O.Box 8730 Beijing 100080 \\ ${ }^{4}$ Department of Modern Physics, Lanzhou University, Lanzhou 730000, P. R. China
}

Using an isospin-dependent quantum molecular dynamics, nuclear stopping in intermediate heavy ion collisions has been studied. The calculation has been done for colliding systems with different neutron-proton ratios in beam energy ranging from $15 \mathrm{MeV} / \mathrm{u}$ to $150 \mathrm{MeV} / \mathrm{u}$. It is found that, in the energy region from above Fermi energy to $150 \mathrm{MeV} / \mathrm{u}$, nuclear stopping is very sensitive to the isospin dependence of in-medium nucleon-nucleon cross section, but insensitive to symmetry potential. From this investigation, we propose that nuclear stopping can be used as a new probe to extract the information on the isospin dependence of in-medium nucleon-nucleon cross section in intermediate energy heavy ion collisions.

PACS number(s): 25.70.Pq, 25.70.-z, 24.10.Lx 
Nuclear stopping in heavy ion collisions (HIC) has been studied by means of rapidity distribution ${ }^{[1]}$ and asymmetry of nucleon momentum distribution ${ }^{[2,3,4]}$. It is an important quantity in determining the outcome of a reaction ${ }^{[5,6]}$. W. Bauer et al. pointed out that in intermediate energy HIC, nuclear stopping power is determined by both the mean field and the in-medium nucleon-nucleon $(\mathrm{N}-\mathrm{N})$ cross section ${ }^{[4,7]}$, but the mean field he used did not include symmetry potential. Recently S. A. Bass, S. J. Yennello, H. Johnston, and Bao-An Li et al suggested that the degree of approaching to isospin equilibration provides a means to probe the mechanism and the power of nuclear stopping in HIC ${ }^{[8-17]}$. But it is not clear how the stopping power depends on the symmetry potential in the same collisions. In this Letter, we report a new possibility to extract information on the in-medium N-N cross section in intermediate energy HIC by using nuclear stopping as a probe. The effects of both in-medium N-N cross section and symmetry potential on nuclear stopping shall be studied comparatively for colliding systems with different neutron-proton ratios in the beam energy ranging from $15 \mathrm{MeV} / \mathrm{u}$ to $150 \mathrm{MeV} / \mathrm{u}$ by using an isospin dependent quantum molecular dynamics (IQMD) model. The calculated results show that stopping power depends strongly on the isospin dependence of the in-medium N-N cross section, but weakly on the symmetry potential for all the colliding systems studied in the beam energy region from $45 \mathrm{MeV} / \mathrm{u}$ to $150 \mathrm{MeV} / \mathrm{u}$. However, in the energy region below Fermi energy, nuclear stopping is sensitive to both the in-medium N-N cross section and the symmetry potential.

The following two quantities can be used to describe nuclear stopping in HIC. The momentum quadrupole $Q_{Z Z}$ defined as $Q_{Z Z}=\sum_{i}^{A}\left(2 P_{z}(i)^{2}-P_{x}(i)^{2}-P_{y}(i)^{2}\right)$, and the transverse-parallel ratio of momentum $R$ given by $R=(2 / \pi)\left(\sum_{i}^{A}\left|P_{\perp}(i)\right|\right) /\left(\sum_{i}^{A}\left|P_{\|}(i)\right|\right)$. Here the total mass $\mathrm{A}$ is the sum of the projectile mass $A_{p}$ and the target mass $A_{t}$. The values of the transverse and parallel components of the momentum of the i-th nucleon are $P_{\perp}(i)=\sqrt{P_{x}(i)^{2}+P_{y}(i)^{2}}$ and $P_{/ /}(i)=P_{z}(i)$, respectively.

In order to describe the isospin effects on the dynamical process of HIC, quantum molecular dynamics $\left(\mathrm{QMD}^{[11]}\right)$ should be modified properly: (1) the density dependent mean field should contain the correct isospin-dependent terms including symmetry potential and Coulomb potential, (2) the in-medium N-N cross section should be different for neutron-neutron ( proton-proton ) and neutron-proton collisions, and finally, (3) Pauli blocking should be counted by distinguishing neutron and proton. The interaction potential is given by

$$
U=U^{S k y}+U^{Y u k}+U^{C o u l}+U^{M D I}+U^{\text {Pauli }}+U^{S y m},
$$


where $U^{S k y}$ is the density-dependent Skyrme potential,

$$
U^{S k y}=\alpha\left(\frac{\rho}{\rho_{0}}\right)+\beta\left(\frac{\rho}{\rho_{0}}\right)^{\gamma}
$$

$\alpha=-390.1 \mathrm{MeV}, \beta=320.3 \mathrm{MeV}$ and $\gamma=1.1667$. $U^{\text {Coul }}$ the Coulomb potential and $U^{Y u k}$ the Yukawa potential (for detail, see Refs.[18]). $U^{M D I}$ is the momentum-dependent interaction ${ }^{[11]}$,

$$
U^{M D I}=t_{4} \ln ^{2}\left[t_{5}\left(\overrightarrow{p_{1}}-\overrightarrow{p_{2}}\right)^{2}+1\right] \frac{\rho}{\rho_{0}}
$$

where the parameters $t_{4}=1.57 \mathrm{MeV}, t_{5}=5 \times 10^{-4} \mathrm{MeV}^{-2}, \rho$ and $\rho_{0}$ are nuclear density and its normal value, respectively. $U^{\text {Pauli }}$ is the Pauli potential,

$$
U^{\text {Pauli }}=V_{p}\left(\frac{\hbar}{p_{0} q_{0}}\right)^{3} \exp \left(-\frac{\left(\overrightarrow{r_{i}}-\overrightarrow{r_{j}}\right)^{2}}{2 q_{0}^{2}}-\frac{\left(\overrightarrow{p_{i}}-\overrightarrow{p_{j}}\right)^{2}}{2 p_{0}^{2}}\right) \delta_{p_{i} p_{j}}
$$

where $\delta_{p_{i} p_{j}}=1$ for neutron-neutron or proton-proton, and $\delta_{p_{i} p_{j}}=0$ for neutron-proton. The parameters $V_{p}=30 \mathrm{MeV}, p_{0}=400 \mathrm{MeV} / \mathrm{c}$ and $q_{0}=5.64 \mathrm{fm}$. According to our experience, the interaction potentials containing Pauli potential can describe the structure effect of fragmentation in the dynamical process of HIC [19].

$U^{s y m}$ is the symmetry potential. In the present calculation, three different density dependences of the symmetry potential ${ }^{[12]}$ are used, i.e., $U_{1}^{\text {sym }}=c F_{1}(u) \delta \tau_{z}, U_{2}^{\text {sym }}=c F_{2}(u)\left[\delta \tau_{z}+\frac{1}{2} \delta^{2}\right]$, and $U_{3}^{\text {sym }}=$ $c F_{3}(u)\left[\delta \tau_{z}-\frac{1}{4} \delta^{2}\right]$, where $\tau_{z}=1$ for neutron and $\tau_{z}=-1$ for proton, $\mathrm{c}$ is the strength of symmetry potential, taking the value of 0 or $32 \mathrm{MeV} . F_{1}(u)=u, F_{2}(u)=u^{2}$ and $F_{3}(u)=u^{1 / 2}, u \equiv \rho / \rho_{0} . \delta$ is the relative neutron excess $\delta=\frac{\rho_{n}-\rho_{p}}{\rho_{n}+\rho_{p}}=\frac{\rho_{n}-\rho_{p}}{\rho}$, where $\rho_{n}$ and $\rho_{p}$ are neutron and proton densities, respectively. First of all, the density distribution of nucleus was calculated by using Skyrme-Hatree-Fock with parameter set SKM*[22].

An empirical expression of the in-medium N-N cross section ${ }^{[20]}$ is used, $\sigma_{N N}^{m e d}=\left(1+\alpha \frac{\rho}{\rho_{0}}\right) \sigma_{N N}^{f r e e}$, with the parameter $\alpha \approx-0.2$, and $\sigma_{N N}^{f r e e}$ is the experimental free $\mathrm{N}-\mathrm{N}$ cross section ${ }^{[21]}$. It is known that the free neutron-proton cross section is about 3 times larger than the free proton-proton or neutron-neutron cross section below $300 \mathrm{MeV}$.

The nuclear stopping are studied for the colliding systems ${ }^{20} \mathrm{Ne}+{ }^{20} \mathrm{Ne},{ }^{40} \mathrm{Ar}+{ }^{40} \mathrm{Ar},{ }^{80} \mathrm{Zn}+{ }^{80} \mathrm{Zn}$, ${ }^{112} S n+{ }^{112} S n$ and ${ }^{124} S n+{ }^{124} S n$ at different beam energies ranging from $15 \mathrm{MeV} / \mathrm{u}$ to $150 \mathrm{MeV} / \mathrm{u}$. The neutron-proton ratios of the above colliding systems are 1.0, 1.22, 1.67, 1.24, and 1.48, respectively. To make a comparative study of the isospin effects of the in-medium N-N cross section and the symmetry potentials, we have investigated the following three different cases. (i) The symmetry potential $U^{\text {sym }}=U_{1}^{\text {sym }}$ is used with the strength $c=32 \mathrm{MeV}$ and the isospin dependent in-medium N-N cross section is employed. This 
case is denoted by $\mathrm{C}=U_{1}^{\text {sym }}+\sigma^{i s o}$ and solid lines in the figures. (ii) $U^{\text {sym }}=U_{1}^{\text {sym }}$ and $\sigma_{N N}^{\text {med }}$ is isospinindependent, denoted by $\mathrm{C}=U_{1}^{\text {sym }}+\sigma^{\text {noiso }}$ and dash lines. (iii) There is no symmetry potential $(c=0)$ and $\sigma_{N N}^{m e d}$ is isospin-dependent, denoted by $\mathrm{C}=0+\sigma^{i s o}$ and dotted lines.

Fig.1 depicts the time evolution of $R$ for central collisions of the systems ${ }^{112} S n+{ }^{112} S n$ (top row), and ${ }^{124} \mathrm{Sn}+{ }^{124} \mathrm{Sn}$ (bottom row), with the beam energies of $15 \mathrm{MeV} / \mathrm{u}, 25 \mathrm{MeV} / \mathrm{u}, 35 \mathrm{MeV} / \mathrm{u}, 45 \mathrm{MeV} / \mathrm{u}, 72 \mathrm{MeV} / \mathrm{u}$, $100 \mathrm{MeV} / \mathrm{u}$, and $150 \mathrm{MeV} / \mathrm{u}$ for the above three cases. It is very clear that for both colliding systems the nuclear stopping $R$ depends strongly on the isospin dependence of the in-medium N-N cross section and weakly on the symmetry potential as the beam energy is above $45 \mathrm{MeV} / \mathrm{u}$. As the beam energy decreases to below the Fermi energy, the nuclear stopping depends on both isospin dependence of in-medium N-N cross section and the symmetry potential.

In Fig. 2, the time evolution of $Q_{Z Z}$ is shown for central collisions of the system ${ }^{112} \mathrm{Sn}+{ }^{112} \mathrm{Sn}$ at different beam energies in the case of $C=U_{1}^{s y m}+\sigma^{i s o}$. It is clearly shown that as decreasing the beam energy, the asymptotic value of $Q_{Z Z}$ decreases towards zero, indicating an isotropic nucleon momentum distribution of the whole composite system and consequently a full stopping at the beam energy below Fermi energy. As beam energy increases to above Fermi energy, because of the pre-equilibrium particle emission and the longitudinal motion of the project-like and target-like nuclei, $Q_{z z}$ will get certain non-vanishing value, indicating partial transparency. It is also seen that the relaxation time decreases as increasing beam energy, which shows the fact that high beam energy leads to more violent $\mathrm{N}-\mathrm{N}$ collisions and faster dissipation. This is consistent with the isospin equilibrium process as shown by Bao-An Li et al. ${ }^{[12]}$.

In order to trace neutron and/or proton observables, in Fig. 3 is given the time evolution of $Q_{z z}$ for neutron and proton respectively. The conclusion drawn from Fig. 1 is applicable to both neutron and proton stoppings.

In Fig. 4 is plotted the time evolution of $R$ for the reaction ${ }^{112} \mathrm{Sn}+{ }^{112} \mathrm{Sn}$ at $E=100 \mathrm{MeV} / \mathrm{u}$ for different impact parameters. It is noticed that the nuclear stopping for small impact parameters $(b=0.0 \mathrm{fm}, 1.0 \mathrm{fm}$, 2.0fm) shows the same behavior as the central collisions as in Fig. 1, while as increasing the impact parameter, the behavior becomes different and the dominant role played by the isospin dependent in-medium N-N cross section gradually disappears. The impact parameter dependence of $R(b)$ can be seen more clearly from Table 1 where the asymptotic values of $R$ are given for different $b$.

Table 1 The asymptotic values of the stopping $\mathrm{R}$ for the reaction ${ }^{112} \mathrm{Sn}+{ }^{112} \mathrm{Sn}$ at $\mathrm{E}=100 \mathrm{MeV} / \mathrm{u}$ as function of impact parameter 


\begin{tabular}{|c|c|c|c|c|c|c|}
\hline $\mathrm{b}(\mathrm{fm})$ & 0.0 & 1.0 & 2.0 & 3.0 & 4.0 & 5.0 \\
\hline$R\left(U_{1}^{\text {sym }}+\sigma^{\text {noiso }}\right)$ & 0.70 & 0.72 & 0.60 & 0.55 & 0.51 & 0.50 \\
\hline$R\left(U_{1}^{\text {sym }}+\sigma^{\text {iso }}\right)$ & 0.82 & 0.82 & 0.71 & 0.63 & 0.56 & 0.50 \\
\hline$R\left(0+\sigma^{i s o}\right)$ & 0.84 & 0.81 & 0.74 & 0.64 & 0.58 & 0.51 \\
\hline
\end{tabular}

In Fig. 5 is shown the impact parameter-averaged asymptotic values ( the values at $t \geq 200 \mathrm{fm} / \mathrm{c}$ when the nuclear stopping becomes nearly a constant as shown in Figs. 1 and 2) of $Q_{z z}$ per nucleon (left window) and $R$ (right window) as a function of $A_{T}+A_{P}$ in the following seven cases: $\mathrm{C}=0+\sigma^{i s o}$ by the solid square; $\mathrm{C}=U_{1}^{\text {sym }}+\sigma^{i s o}$ denoted by the solid circles; $\mathrm{C}=U_{2}^{\text {sym }}+\sigma^{\text {iso }}$ by solid triangle; $\mathrm{C}=U_{3}^{\text {sym }}+\sigma^{\text {iso }}$ by solid diamond; $\mathrm{C}=U_{1}^{\text {sym }}+\sigma^{\text {noiso }}$ by open circles; $\mathrm{C}=U_{2}^{\text {sym }}+\sigma^{\text {noiso }}$ by open triangle; $\mathrm{C}=U_{3}^{\text {sym }}+\sigma^{\text {noiso }}$ by open diamond. In the calculation the beam energy is $100 \mathrm{MeV} / \mathrm{u}$ and five colliding systems have been considered, i.e., ${ }^{20} \mathrm{Ne}+{ }^{20} \mathrm{Ne},{ }^{40} \mathrm{Ar}+{ }^{40} \mathrm{Ar},{ }^{80} \mathrm{Zn}+{ }^{80} \mathrm{Zn},{ }^{112} \mathrm{Sn}+{ }^{112} \mathrm{Sn}$ and ${ }^{124} \mathrm{Sn}+{ }^{124} \mathrm{Sn}$. From Fig. 5, it can be seen that the nuclear stopping depends strongly on the isospin dependence of in-medium N-N cross section and weakly on the symmetry potential, though it is slightly different for the three different forms of symmetry potential, especially for larger-mass systems. It is also shown that the nuclear stopping power increases as increasing the total mass of the colliding system, the decrease of $Q_{z z}$ or the increase of $R$ implies the increase of nuclear stopping.

Fig. 6 shows the asymptotic value at $t=200 \mathrm{fm} / \mathrm{c}$ of $R$ as a function of the beam energy for the same colliding systems and the same collision conditions as in Fig. 1. From the figure, one can draw the same conclusion as from Fig. 1.

From the above comparative study of nuclear stopping for different colliding systems, beam energies, and impact parameters by using IQMD, we have obtained the following physical picture of the collision dynamics: from above the Fermi energy to about $150 \mathrm{MeV} / \mathrm{u}$, the dynamics is dominated by N-N collisions and the role played by the mean field is less important. The main consequence of the N-N collisions is the transformation of the initial longitudinal motion to the motion in other directions and the subsequent thermalization of the system. In this case, nuclear stopping $R$ and $Q_{z z}$ are sensitive to nucleon-nucleon collisions, and can thus be used as a measure of the dissipative process. However, as the beam energy decreases to below the Fermi energy, the collision dynamics is governed by both the mean field and the N-N collision which result in one-body and two-body dissipation or thermalization, respectively. Thus as indicators of the thermalization, $R$ and $Q_{z z}$ depend on both the mean field and the in-medium N-N cross-section.

In summary, from the above results and discussions we conclude that the nuclear stopping $R$ and $Q_{z z}$ can be used as a probe to extract information on the isospin dependence of the in-medium N-N cross section in $\mathrm{HIC}$ in the beam energy region from above the Fermi energy to about $150 \mathrm{MeV} / \mathrm{u}$. 


\section{Acknowledgment}

This work was supported in part by the " 100 person project" of the Chinese Academy of Sciences, National 973 subject under grant No. G2000077400, the National Natural Foundation of China under Grants No. 19775057 and No. 19775020, No. 19847002, No. 19775052 and KJ951-A1-410, by the Foundation of the Chinese Academy of Sciences.

\section{References}

[1] C.Y. Wong, Introduction to High-Energy Heavy-Ion Collisions, World Scientific (Singapore), 1994.

[2] B.A. Li and C. M. Ko, Nucl. Phys. A601, 457(1996).

[3] F. Videbaeck and O. Hansen, Phys. Rev. C52, 2684(1995)

[4] W. Bauer, Phys. Rev. Lett. 61, 2534(1998).

[5] W. Busza and R. J. Ledoux, Annual Review of Nuclear and Particle Science Vol.38, 119(1988).

[6] B. A. Li and C. Y. Wong, Phys. Scr V47, 151(1993).

[7] G. F. Bertsch, G. E. Brown, V. Koch and B. A. Li, Nucl. Phys. A490, 745(1988).

[8] S. A. Bass, J. Konopka, M. Bleicher, H. Stöcker and W. Greiner, GSI annual report P.66 (1994).

[9] B. A. Li and S. J. Yennello, Phys. Rev. C52, R1746(1995).

[10] H. Johnston, T. Wigner, T. White, B. Harst, D. O'kelly and S. J. Yennello, Phys. Lett. B371, 186(1996).

[11] G. Peilert, A. Rosenhauer, J. Aichelin, H. Stöcker and W. Greiner, Phys. Rev. C39, 1402(1989).

[12] B. A. Li, C. M. Ko and W. Bauer, Inter. Jour. Mod. Phys. E Vol.7, No.2, 147-229(1998).

[13] A. Ono and H. Horiuchi, Phys. Rev. C53, 2958(1996).

[14] M. Farine, T. Sami, B. Remand and F. Sebille, Z. Phys. A339, 363(1991).

[15] H. Johnston, T. White, B. A. Li, E. Ramakrishman, J. Winger, D. J. Rowland, B. Hurst, F. GimenoNogues and D. O'Kelly, Phys. Rev. C56, 1972(1997).

[16] S. J. Yennello, B. Young, J. Yee, J. A. Wigner et al., Phys. Lett. B321, 15(1994).

[17] H. Johnston, J. Wigner et al., Phys. Lett. B371, 186(1996). 
[18] J. Aichelin, G. Peilert, A. Bohnet, A. Rosenhauer, H. Stöcker and W. Greiner, Phys. Rev. C37, 2451(1988).

[19] Liu Hang, Liu Jianye, Li Junqing, Z. Physik. A354, 311(1996).

[20] D. Klakow, G. Wilke and W. Bauer, Phys. Rev. C48, 1982(1993).

[21] K. Chen, Z. Fraenkel, G. Friedlander, J. R. Grover, J. M. Miller and Y. Shimamoto, Phys. Rev. 166, 949(1968).

[22] P.G. Reinhard, in Computational Nuclear Physics 1, editted by K. Langanke, J.A. Maruhn, and S.E. Koonin, Germany, Springer-Verlag, 1991, P.28-50 


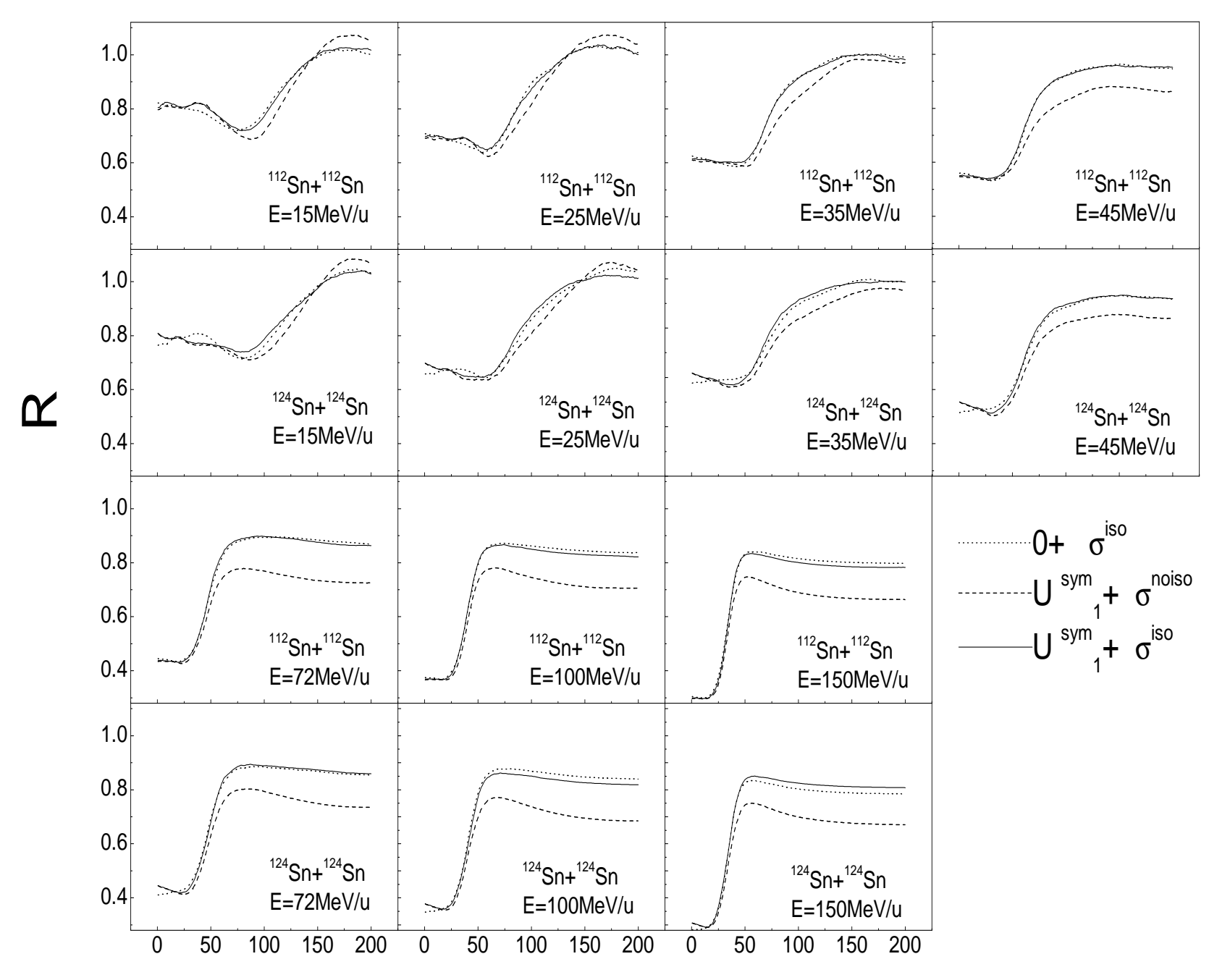

\section{Time $(\mathrm{fm} / \mathrm{c})$}




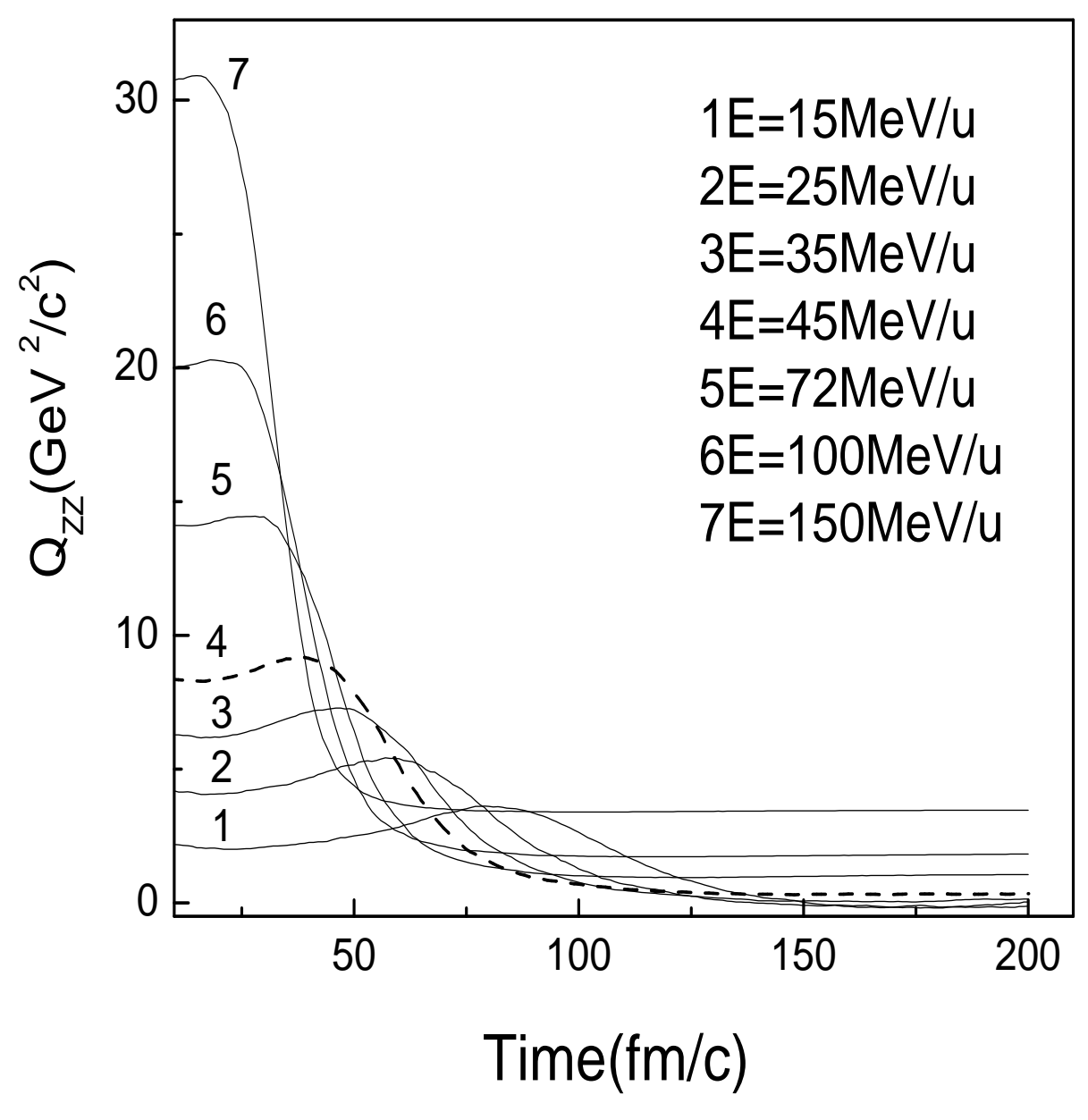




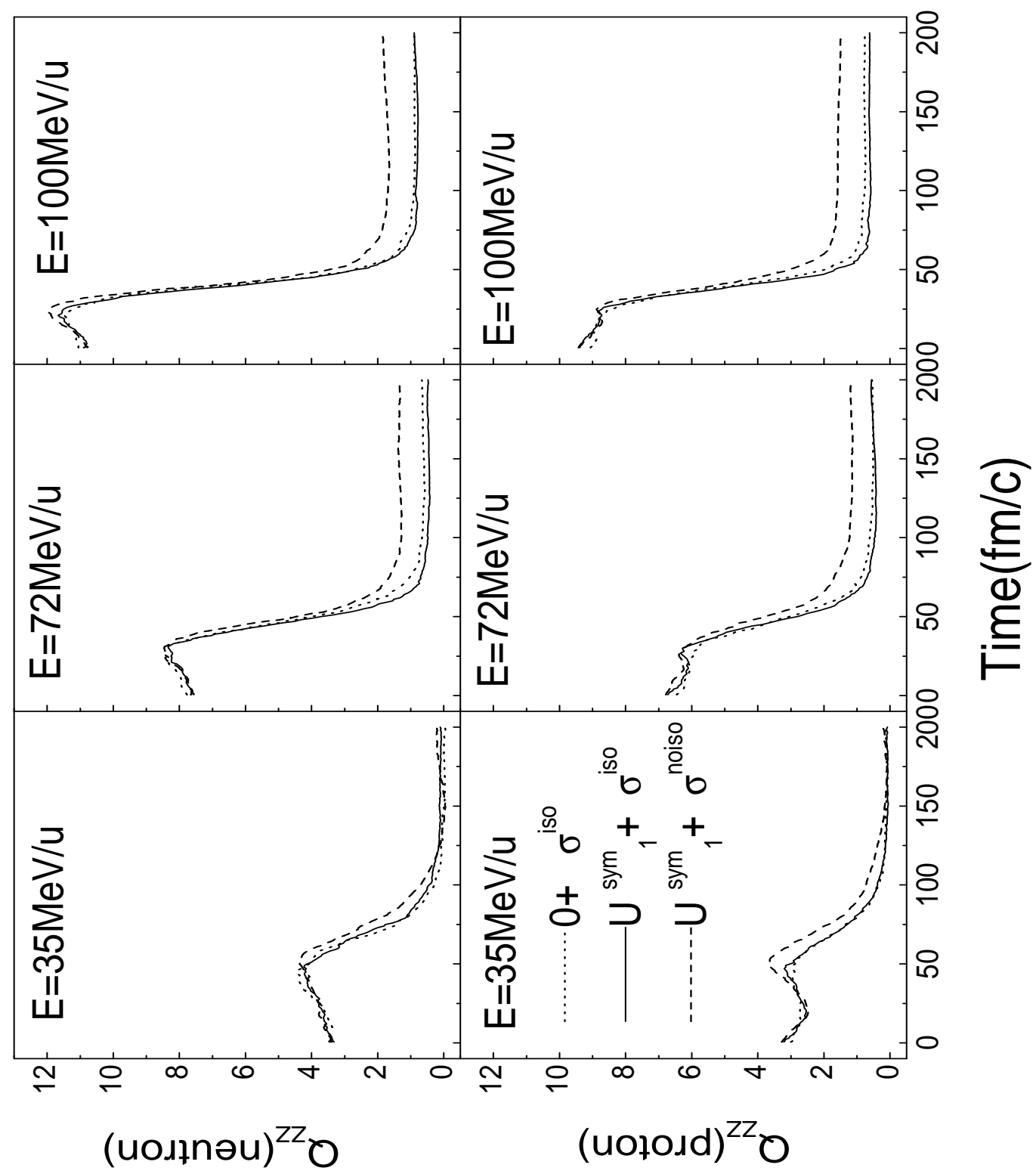

Figure 3: The time evolution of momentum quadrupoles for neutron $Q_{z z}$ (neutron) and proton $Q_{z z}$ (proton) for the central collisions of ${ }^{112} \mathrm{Sn}+{ }^{112} \mathrm{Sn}$ at the beam energies of $35 \mathrm{MeV} / \mathrm{u}, 72 \mathrm{MeV} / \mathrm{u}$, and $100 \mathrm{MeV} / \mathrm{u}$ in the three cases as shown in Fig.1. 


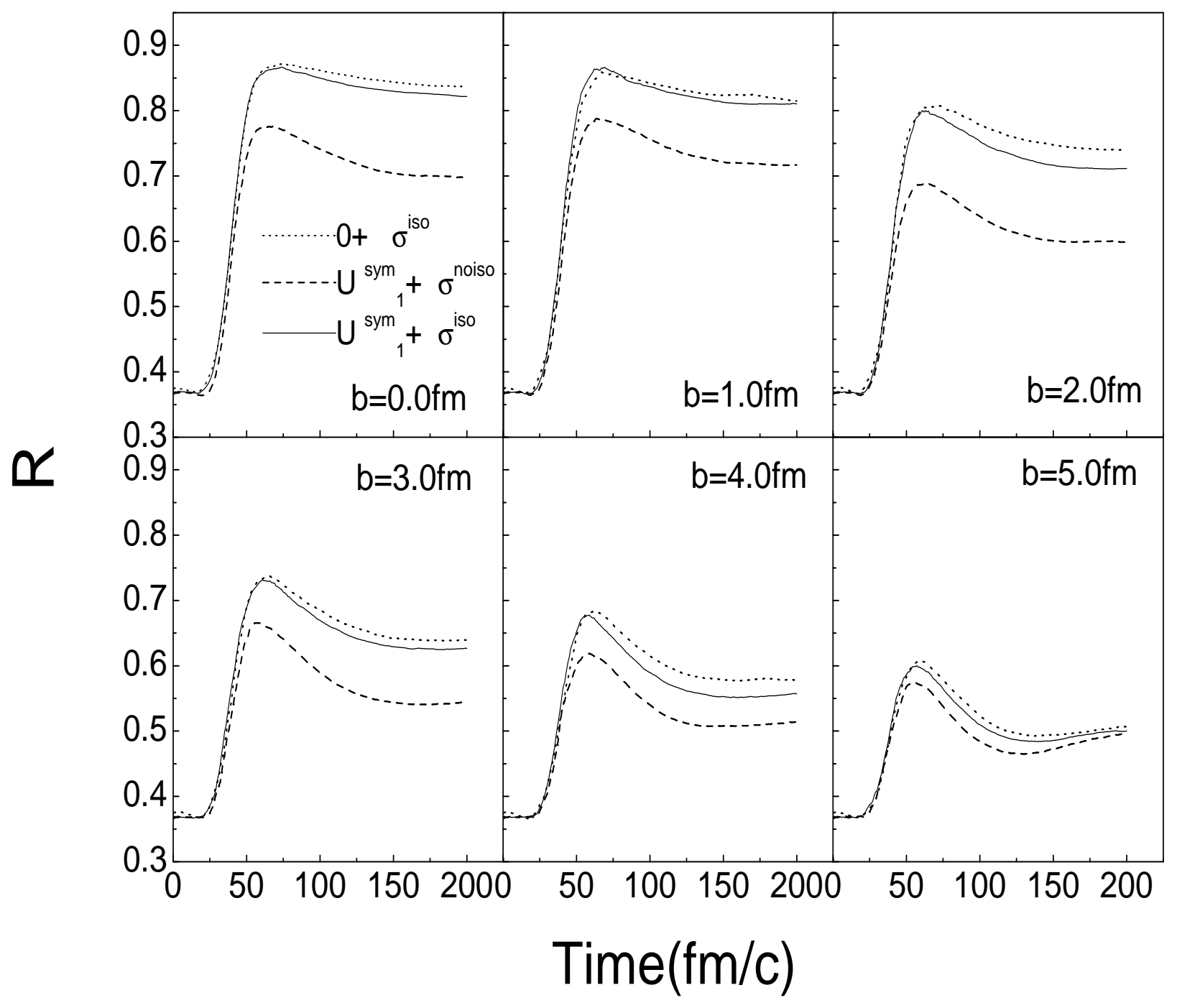




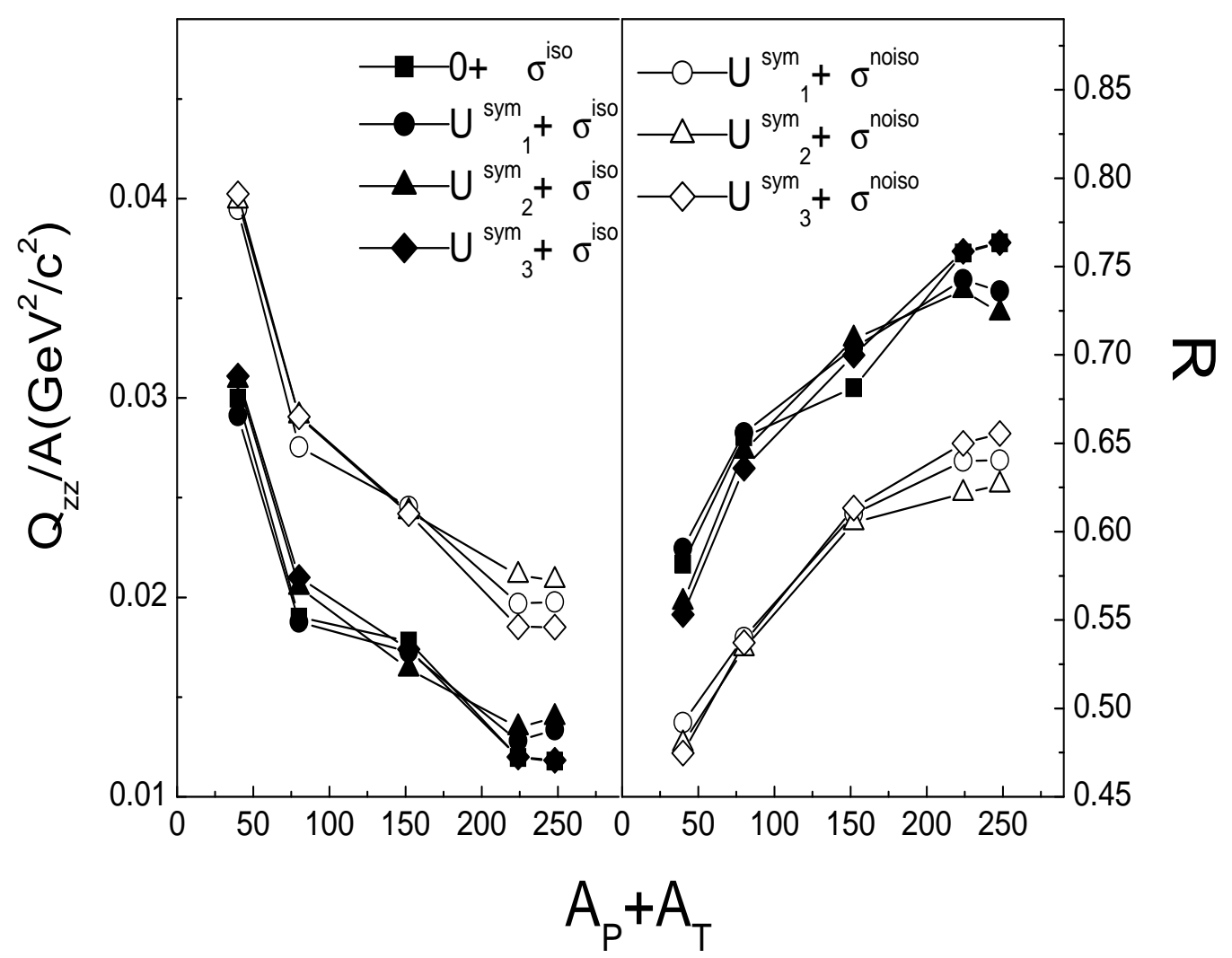




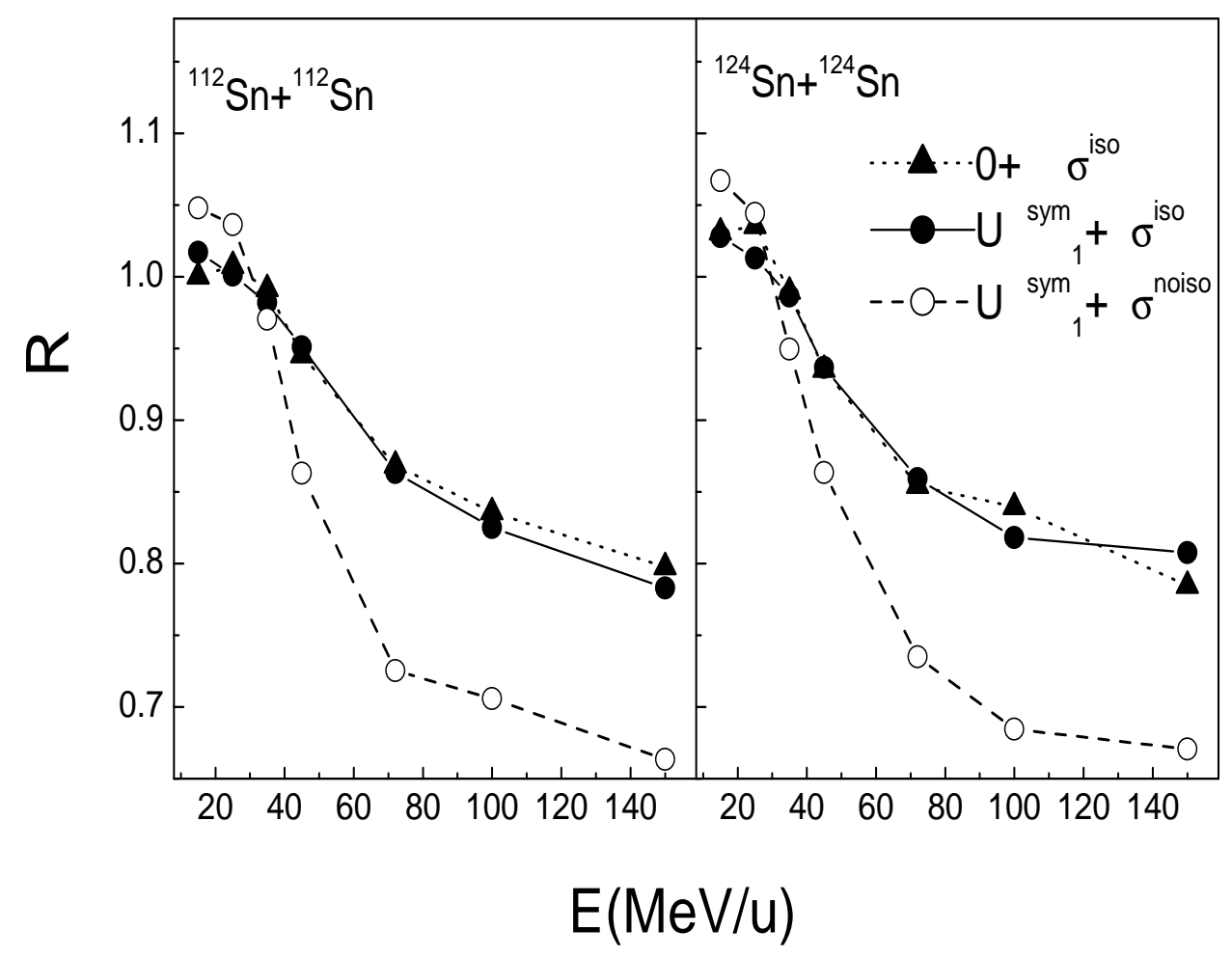

